To list an event in the Calendar, contact J. Dininny, Materials Research Society, 9800 McKnight Road, Pittsburgh, PA 15237; (412) 367-3036; $\operatorname{tax}(412)$ 367-4373.

MIRS identifies meetings sponsored or co-sponsored by the Materials Research Society; boldface type without the logo identifies MRS-endorsed meetings.

See MRS BULLETIN Vol. XV No. 1 for Calendar events from February 1 through February 28, 1990.

\section{MARCH 1990}

4-9

Symposium on Microlithography San Jose, CA

SPIE, P.O. Box 10, Bellingham WA 98227-0010; (206) 676-3290; $\operatorname{tax}(206) 647-1445$

5-9

41st Pittsburgh Conference on Analytical Chemistry and Applied Spectroscopy

New York, NY

A. Johnson, Pittsburgh Conference, 300 Penn Ctr. Blvd., Suite 332, Pittsburgh, PA 15235

5-9

Gordon Research Conterence on Nondestructive Evaluation Ventura, CA

A.M. Cruickshank, Gordon Research Conf., Univ. of Rhode Island, Kingston, RI 02881-0801: (401) 783-4011; $\operatorname{tax}(401)$ $783-7644$

$6-8$ 2nd Symposium on Integrated Ferroelectrics Monterey, CA

A.S. Miller, Microelectronics Research Labs., Univ. of Colorado at Colorado Springs, P.O. Box 7150: Colorado Springs, CO 80933-7150; (719) 593-3488

7

Physics and Chemistry of Uttrathin Films

Piscataway, $N J$

J. Thomas, Greater New York Chapter of the AVS, David Samoff Research Center, Princeton, NJ 08543-5300; (609) 734-3243

$10-11$

Conducting Polymers: Electronic and Optical Properties

Anaheim, CA

J. Spellos, American Physical

Society, 335 E. 45th St. New

York, NY 10017; (212) 682-7341
$12-16$

Gordon Research Conference on Crystal Growth

Oxnard, CA

A.M. Cruickshank, Gordon

Research Conf., Univ. of Rhode

Island, Kingston, RI 02881-0801;

(401) 783-4011; $\operatorname{tax}(401)$ 783-7644

\section{$12-16$}

American Physical Society Meeting Anaheim, CA

W.W. Havens Jt. APS, $335 \mathrm{E}$

45th St. New York, NY 10017;

(212) $682-7341$

12-30

Experimental Workshop on HighTemperature Superconductors and Related Materials

Trieste, Italy

Intemational Center for Theoreti-

cal Physics, HTS Experimental

Workshop (BASIC), P.0. Box 586,

1.34100 Trieste, Italy

17.21

Symposium on Advances in

Semiconductors and Super-

conductors

San Diego, CA

SPIE, P.O. Box 10, Bellingham,

WA 98227.0010; (206) 676-3290

19-21

9th International Conference on

Thermoelectrics

Pasadena, CA

A. Finnin, MS 277-212, Je

Propulsion Lab., 4800 0ak Grove

Dr., Pasadena, CA 91109; (818)

354-3223; $\operatorname{tax}(818)$ 393-6951

$19-22$

U.K. Intormation Technology

Conference

Southampton University, United

Kingdom

IEE, Conterence Services, Savo

Place, London WC2R OBL - 01-

240 1871; $\operatorname{tax} 01-240-7735$

19-23

Gordon Research Conference on

Superconductivity

Ventura, CA

A.M. Cruickshank, Gordon

Research Conf., Univ. of Rhode

Island, Kingston, RI 02881-0801

(401) 783-4011; $\operatorname{tax}(401)$

$783-7644$

19-23

Gordon Research Conference on

Polymers in Biosystems

Oxnard, CA

A.M. Cruickshank, Gordon

Research Conf., Univ. of Rhode

Island, Kingston. RI 02881-0801

(401) 783-4011; tax (401)

$783-7644$

25-27

1st Joint Technology Conterence

San Diego, CA

ISHM JTC 1990, P.O. Box 2698

Reston, VA 22090; (703) 471

0066 ; $\operatorname{tax}(703)$ 471-1937
26-29

Space Commerce ' 90

Montreux, Swizzerland

Secretariat, Space Commerce ' 90

P.0. Box 97, CH-1820 Montreux,

Swizerland; + 41219632354

$\operatorname{tax}+41219637895$

26-30

Gordon Research Conference on

Thrombolysis

Ventura, CA

A.M. Cruickshank, Gordon

Research Cont Univ of Rhode

Island, Kingston, RI 02881-0801

(401) 783-4011; $\operatorname{tax}$ (401)

$783-7644$

\section{APRIL 1990}

$1-6$

8th International Conference on

Thin Films, in conjunction with

Intemational Conference on

Metallurgical Coatings

San Diego, CA

WD. Sproul Bas Ind Res Lab

Norttwestern Univ., 1801 Maple

Ave., Evanston, IL 60201-3135;

(312) 491-4108; $\operatorname{tax}(312)$

$491-4486$

$1-7$

1st Intemational Conference on

Epitaxial Cystal Growth

Budapest, Hungary

E. Lendvay, Res. Inst. for Tech.

Phys., Hungarian Acad. of Sci.

Uipest 1, Pf 76, Budapest.

Hungary-1325; tax (36) (1)

698-037

2-6

Intemational Congress on Optical

Science and Engineering

Hamburg. W. Germany

SPIE, P.0. Box 10, Bellingham,

WA 98227-0010; (206) 676-3290; $\operatorname{tax}(206)$ 647-1445

$2-6$

Advances in Materials Science and Applications of High-Temperature

Superconductors

Greenbelt, MD

Y. Flom, NASA Goddard Space

Flight Center, Code 313.0, Bldg.

22. Rm. 339 , Greenbelt, MD

20771; (301) 286-3274

3

3nd Annual Buffalo Symposium

on Materials and Processes

Buffalo, NY

C. Lorge, Wilson Grearbatch Ltd. 10,000 Wehrte Drive, Clarence. NY 14031; (716) 959-6901

4-6

Fine Ceramic Powders

University of Warwick, United

Kingdom

Institute of Ceramics, Shelton

House, Stoke Rd., Shelton,

Stoke-on-Trent ST4 2DR; (0782)

202116: $\operatorname{tax}$ (0782) 202421
Physics of Advanced Materials Systems

Alfred NY

E.C. Behrman, New York State

Section of the American Physical

Society, Alfred Univ, Alfred, NY

14802; (607) 871-2249

8.13

American Crystallographic

Association Meeting

New Orleans, LA

ACA, P.0. Box 96, Ellicott Station,

Butfalo, NY 14025-0096; (716)

$856-9600$, ext. 321

$10-12$

New Materials and Their

Applications

Warwick, United Kingdom

Institute of Physics, 47 Belgrave

Square, London SW1X 80X,

United Kingdom; 01-235 6111;

tax 01-259 6002

$12-13$

3rd International Conterence on

Amorphous and Crystalline

Silicon Garbide and other Group

IV-IV Materials

Washington, DC

G.L. Harris, Materials Science

Research Ctr. of Excellence,

Howard Univ./School of Engineer-

ing, 2300 Sixth St. NW, Washing.

(202) 636-6618;

$\operatorname{fax}(202) 636.5960$

16-20

Florida Catalysis Conference

Palm Coast, FL

R.S. Drago, Dept. of Chemistry

Univ of Florida Gainesville FL 32611

16-21

\section{MIRS Materials Research}

Society Spring Meeting

San Francisco, CA

M. Geil, Matenials Research

Society, 9800 McKnight Rd.

Pittsburgh, PA 15237; (412) 367.

3003; $\operatorname{tax}(412) 367-4373$

22-26

ACerS 92nd Annual Meeting

Dallas, TX

American Ceramic Society, 757

Brooksedge Plaza Dr., Westerville

OH 43081; (614) 890-4700

22-27

American Chemical Society

Meeting

Boston, MA

American Chemical Society, 1155

16th St. NW. Washington, DC

20036; (202) 872-4600

23-25

Material Aspects of Machining

Brugge, Belgium

R. Peys, Ingenieurshuis vaw,

Desguinlei 214, B-2018 Antwer-

pen; 03/21609 96;

$\operatorname{tax} 03 / 2160689$

$33-25$

2nd Intemational Conference on Indium Phosphide and Related

Materials

Denver, CO

Lasers and Electro-0ptics Society,

445 Hoes Lane, P.O. Box 1331

Piscataway, NJ 08855-1331; (201)

562-3895; tax (201) 981-0027

23-26

Intemational Conference on Low

Temperature Electronics

Berkeley CA 
29-4

International Conterence on

Transport Properties of Super-

conductors

Rio de Janeimo, Brazil

R. Nicolsky, Instituto de Física,

Universidade Federal do Rio de

Janeiro, C.P. 68528, 21945 Rio de

Janeiro, Brazil; (55) (21) 270-1191

270-1736; $\operatorname{tax}$ (55) (21) 280-7693

30-4

Conterence on the Science and

Technology of Thin Film Super-

conductors

Denver, CO

D. Christodaro, Solar Energy

Research Institute, Conf.,

Coordination Section, 1617 Cole

Blvd. Golden CO 80401-3393.

(303) 231-1158; $\operatorname{tax}$ (303) 231-1199

\section{MAY 1990}

3

Advanced Materials for the 90 s

Washington, DC

TMS, 420 Commonwealth $\mathrm{Dr}$.

Wasrendale, PA 15086; (412) 776

9050; $\operatorname{tax}(412) 776-3770$

$6-8$

Symposium on High $T_{c}$ Super-

conductor Technologies at the

177 th Electrochemical Society

Meeting

Montreal, Canada

S. Raider, IBM T.J. Watson

Research Ctr. P.O. Box 218,

Yorktown Heights, NY 10598 ;

(914) $945-2203$

6-11

177th Electrochemical Society

Meeting

Montreal, Canada

Electrochemical Society, $10 \mathrm{~S}$

Main St., Pennington, N 08534

2896; (609) 737-1902

$9-11$

ICMC-90: High Temperature

Superconductors-Materials

Aspects

Garmisch-Partenkirschen,

W. Germany

Conference Secretariat: ICMC-

90-HTSC, Deutsche Gesell-

schaft für Metallkunde, eV,

Adenauerallee 21, D-6370

Obenursel, W. Germany; (06171)

4081, tax (06171) 52554 .

13-16

6 th Conference on Semi-

Insulating III-V Materials

Tomnto, Canada

C. Miner, Bell-Northern Research,

P.0. Box 3511, Station C, Ottawa,

Ontario K1Y 4H7; tax (613) 763-

2404
13-25

Intemational School on Cystal

Growth and Crystallographic

Assessment of Industria

Materials

Barcelona, Spain

R. Rodríguez-Clemente, Institute

of Materials Science, C.S.I.C.-c

Marti i Franqués, sin 08028

Barcelona, Spain; 34-3-3302716; tax 34-3-4110012

14-16

Computer-Assisted Image

Analysis and Measurement

Ra!eigh, NC

B. Winston, Dept. of Lifelong Ed.,

N.C. State Univ., Raleigh, NC

27695; (919) 737-2261

15-18

Intemational Conference on

Dynamics of Flexible Structures in

Space-1990

Cranfield, Bedford, United

Kingdom

Conference Sec. College of

Aeronautics, Cranfield Institute of

Technology, Cranfield, Bedford

MK43 OAL, United Kingdom

0234 750111, ext. 2744: tax

(0234) 751550

17-18

Nonmetallic Materials and

Composites at Low Temperatures

Heidelberg, W. Germany

G. Hartwig, Kemforschungszen-

trum Karlsnuhe, Institut fur

Materials- und Festkorperior-

schung N Posttach 3640 0.7500

Kartsruhe, W. Germany; (07247)

823789; tax (07247) 825070

20-24

Frontiers of Electron Microscopy

in Materials Science

Oak Brook, IL

W.E. King, Chemistry and

Materials Science, Dept. L-356,

Lawrence Livermore National

Laboratory, Livermore, CA 94550:

(415) 423-6547; tax (415) 423-

7040

$20-24$

International Conterence on

Organic Superconductors

S. Lake Tahoe, CA

W.A. Little, Physics Dept.

Stanford Univ., Stantord CA

94305-2196; (415) 723-4233

20-24

Workshop on the Science and

Technology of Diamond Thin

Films

Concord, $\mathrm{OH}$

J.C. Angus, Chemical Engrg

Dept., Case Westem Reserve

Univ., Cleveland, $\mathrm{OH} 44106$;

(216) 368-4133; tax (216)

368-3016

(See related article in

Vol. XV No. 11.)
21-25

CLEOAQEC-Conterence on

Lasers and Electro-Optics'

Intemational Quantum Electronics

Conterence

Anaheim, CA

Lasers and Electro-Optics Society,

445 Hoes Lane, P.0. Box 1331 ,

Piscataway, NJ 08855-1331: (201)

562-3895; tax (201) 981-002

21-25

NETWORKS 90: 10th Polymer

Networks Group Meeting and

IUPAC 10th International Sympo

sum on Polymer Networks

Jerusalem, Israel

M. Gottlieb, Chemical Engineering

Dept., Ben Gurion Univ., Beer

Sheva 84105, Israel; 972-57-

461486; fax 972-57-31340

23-25

Fourth Micon Symposium on

Advances in Video Technology:

Materials Science Applications

San Francisco, CA

ASTM, 1916 Race St., Philadel phia, PA 19103

29.31

11 th Intemational SAMPE

European Chapter Conference

Basil, Switzertand

N. Hawley, 1055 W. San Bemar

dino Rd., Covina, CA 91722;

(818) 331-0616

$29-1$

E-MRS European Materials

Research Society Meeting

Strasbourg. France

P. Siffert, Centre de Recherches

Nucleaires, Latromitoire PHASE.

67037 Strasbourn, Cedex,

France; (88) 2865 43;

$\operatorname{tax}(88) 280990$

\section{JUNE 1990}

4-5

9th Annual Symposium on

Electronics Materials, Processing,

and Characterization

Richardson, DX

M.E. Weber, 9th Annual Sympo-

sium, P.0. Box 830914 , Richand-

son, TX 75083-0914; (214)

995-5983

4-6

Optical Spectroscopic Instrument tation and Techniques for the

1990s: Applications in Astronomy,

Chemistry, and Physics

Las Cruces, NM

SPIE, P.O. Box 10 Bellingham,

WA 98227-0010; (206) 676-3290;

$\operatorname{tax}(206)$ 647-1445

$4-8$

Florida Advanced Materials

Chemistry Conterence

Palm Coast, FL

R.S. Drago, Dept. of Chemistry,

Univ. of Florida, Gainesville, FL

32611
$5-7$

4th International Conference on

Advanced infrared Detectors and

Systems

London, United Kingdom

Institution of Electrical Engineers,

Savoy Place, London WC2R OB!

United Kingdom; 01-240 1871

ext. 222; fax 01-240 7735

6-8

High Pertermance Composites

\section{the 19sis}

Morristown, NJ

TMS, 420 Commonwealth Dr.

Warrendale, PA 15085; (412) 776

9050; $\operatorname{tax}(412) 776-3770$

10-13

The Science Behind Materials

Synthesis

University Park, PA

K. Mourant, SMS Conterence.

102 Matenials Research Labor

tory. Pennsytvania State Univer-

sity, University Park, PA

16802-4801; (814) 865-1137

10-18

1st European East-West Sympo-

sium on Materials and Processes

with High Industrial Potential

Helsink, Finland

P. Attila, Teknolink Oy, Hietz-

landenkatu 2B, SF-00180 Hetsinks.

Finland; + 358-06801505; tax

$+358-0-6801599$

11-13

5 th Intemational Workshop on

Electroluminescence

Helsink, Fintand

M. Leskelä, Dept. of Chemical

Engrg. Hetsinki Univ. of Tech.

SF-O2150 Espon, Finland; 3580

451 2597; tax 358-0-462 373

11-14

4th Intemational Symposium on

Nondestructive Characterization

of Materials

Annapolis, MD

R.E. Green Jr., Johns Hopkins

University, Center for NDE,

Maryland Hall, Room 100

Baltimore, MD 21218; (301) 338 7126

11-14

11th Biennial Conterence on

National Materials Policy

Wiliamsburg, VA

B. Houston Federation of

Materials Societies, $1707 \mathrm{~L} \mathrm{St}$

NW, Suite 333, Washington, DC

20036; (202) 296-9282

11-15

Basic Course: SEM and X-Ray

Microanatysis

Bethlehem PA

J.I. Goldstein, Dept. of Materials

Science and Engineering, Bldg. 5

Lehigh University, Bethlehem. PA

18015; (215) 758-5133

$11-15$

10th Intemational Conference on

vacuum Metallurgy

Beīing, China

T. Shaojie, Chinese Society of

Metals, 46 Dongsixi Dajie, Beijing

100711, China: $\operatorname{tax} 5124122$

$12 \cdot 14$

4th Intemational Electronic

Materials and Processes

Conference

Albuquerque, NM

SAMPE, P.0. Box 2459, Covina.

CA 91722; (818) 331-0616

$\operatorname{tax}(818) 332-6929$

13-15 
27-29

32nd Electronic Materials

Conference

Santa Bartara, CA

TMS, Meetings Dept., 420

Commonweatth Dr. Wartendale.

PA 15086; (412) 776-9050; tax

(412) $776-3770$

\section{JULY 1990}

2-5

7th CIMTEC Satellite Symposium

4. High-T $\mathrm{T}_{c}$ Superconductors

(See June 2430 CIMTEC listing)

Trieste, Italy

7th CIMTEC, Satellite Symposium

4. P.0. Box 174, 48018

Faenza, Italy; 546-664143

$\operatorname{tax} 546-664138$

8-13

International Symposium on

Optical and Optoelectronic

Applied Science and Engineering

San Diego, CA

SPIE, P.O. Box 10, Bellingham,

WA 98227-0010; (206) 676-3290;

$\operatorname{tax}(206) 647.1445$

9-12

Conference on Nondestructive

Evaluation of Modem Ceramics

Columbus, $\mathrm{OH}$

American Ceramic Society, 757

8rooksedge Plaza Drive, Wester-

ville, OH 43081; (614) 890-4700;

tax (614) $899-6109$

15-20

8th American Conterence on

Crystal Growth

Vail $\infty$

B.L. Sopori, Solar Energy

Research Institute, 1617 Cole

Bivd., Golden, CO 80401-3393;

(303) 231-1383

17-19

Low Temperature Engineering and

Cryogenics Conterence, LTEC'90

Southampton, England

Cryogenics Conterence Office.

Institute of Cryogenics, University

of Southampton, SOS $5 \mathrm{NU}$,

United Kingdom; 0703-595000

ext. 2059 or 2662; tax 0703

593939

22-27

13th Intemational Liquid Crysta

Conference

Vancouver Canada

B. Bergersen, Dept. of Physics.

Univ. of British Columbia,

Vancouver, V6T 2A6 Canada;

(604) 228-2603

22-3

NATO Advanced Study Institute on Diamond and Diamond-Like Films

Castelvecchio Pascoit, Italy

C.J. McKargue, Metals and

Ceramics Div., Oak Ridge Nationa

Lab. P.O. Box 2008, Oak Ridge,

TN 37831-6118; (615) 574-4344;

$\operatorname{tax}(615) 5747659$
23-26

Conference on Advances in

Cementitious Materials

Gaithersburg, MD

American Ceramic Society, 757

Brooksedge Plaza Drive, Wester-

ville, OH 43081; (614) 890-4700;

$\operatorname{tax}(614) 899-6109$

23-27

5th Intemational Conference on

Scanning Tunneling Microscopy/

Spectroscopy and ist Interna

tional Conference on Nanometer

Scale Science \& Technology

Baltimore MD

J.S. Murday, Code 610Q Naval

Research Lab., Washington, DC

20375-5000; (202) 767-3026; tax (202) 4047139

24-26

Conference on Polymer Blends

Cambridge, United Kingdom

D.C. Varley, Conf. Mgr, PRI, 11

Hobart Place, London

SWIW OHL

$29-3$

37th Intemational Field Emission

Symposium

Albuquerque, NM

G.L. Kellogg, Div. 1114, Sandia

National Laboratories, Albuquer-

que, NM 87185

$30-1$

Pouder Metallurgy: Key to

Advanced Materiads Technology

Vancouver, Canada

Member/Customer Service

Center. ASM Intemational, Metals

Park OH 44073; (216) 338-5151;

$\operatorname{tax}(216) 338-4634$

30-1

POLYSE'90: Polycrystaline Semi conductors

Schwäbisch Hall, W. Germany

H.P. Strunk, Max-Planck Institute

FKF, Heisenbergstr. 1, 0-7000

Stuttgart 8Q FRG; (0711) 6860-

645; $\operatorname{tax}(0711) 6874-371$

$30-2$

8th Intemational Conference

on Ion Implantation Technology

University of Sumey, United

Kingdom

P.L.P. Hemment, Dept of

Electronic \& Electrical Engineer-

ing, Univ. of Surrey, Guildford,

Surrey GU2 5XH; (0483) 571281;

$\operatorname{tax}(0483) 300803$

30-3

5th Intemational Conference on

the Physics of Electro-Optic

Microstructures and Microdevices

Heraldion Crete, Greece

A. Christou, Code 6830 Nava

Research Laboratory, 4555

Overlook Ave. SW, Washington,

DC 20375-5000; $\operatorname{tax}(202)$

$767-0546$
$31-2$

4th International Conference on

Shallow Impurities in Semicon-

ductors

London, United Kingdom

G. Davies, Physics Department

Kings Coltege London, The

Strand, London WC2R $2 L S$

United Kingdom

\section{AUGUST 1990}

8-10

Speciality Polymers ' 90

Baltimore, MD

W. Harvey, SP '90, Butterworth

Scientitic Ltd., P.O. Box 63,

Westbury House, Bury St.,

Guilfford, Surrey, GU2 5BH

United Kingdom; 0483300966 ;

tax 0483301563

$12-18$

12th Intemational Congress for

Electron Microscopy

Seattle, WA

R.M. Fisher, Univ. of Washington,

5001 25th Ave. NE, GH-22.

Seattle, WA 98195; fax (206)

545-9350

13-15

LF-19 Satellite Conference on High

Temperature Superconductivity

Cambridge, United Kingdom

K.S. Diffey, IRC in Super-

conductivity, Madingley Rd.

Cambridge C83 OHE, UK; (0223)

337472; $\operatorname{tax}(0223) 337074$

13-17

7th Intemational Conference on

Rapidly Quenched Materials

Stocktolm, Sweden

Dept. of Casting of Metals, Royal

Institute of Technology, S-100 44

Stockholm, Sweden; + 46-8-790

7984; tax + 46-8-109199

16-21

International Conference-

Chemistry of Electronic Ceramic

Materials

Targhee National Forest, W

R.S. Roth, NIST, Ceramics Div.

420. Bidg. 223, Rm. B216,

Gaitherstourg, MD 20899; (301)

975-6116; fax (301) 975-2128

$26-31$

Light, X-Ray and Neutron

Scattering and Reflectivity from

Polymers: American Chemical

Society National Meeting

Washington, D.C

P.M. Cotts, K93/802, IBM

Almaden Research Ctr. 650

Hamy Rd. San Jose, CA 95120

6099; (408) 927-1638

$27-30$

10th International Symposium on

Boron, Bonides, and Related

Compounds

Albuquerque, NM

T.L. Aselage, Div. 1842, Sandia

National Labs., Albuquerque, NM

87185-5800; (505) 844-0949
27-31

6th Trieste Semiconductor

Symposium: Hydrogen in Semp-

conductors

Trieste, Italy

M. Stutumann, Max Planck

Institut fur Festlopertorschung,

Heisenbergstrasse 1, 0-7000

Stuttgart 80 W. Germany;

(0711)68 60-1; $\operatorname{tax}(0711) 6874371$

27-31

6th International Conference on

Molecular Beam Epitaxy

Lalolla CA

C.W. Tu, Dept. of Elect. and

Comp. Engineering, MCR-007,

Univ. of Caiff. San Diego, La

Jolla, CA 92003-0407; (619) 534

4687; tax (619) 534-2486

2831

Intemational Conference on

Computer Applications to Materials

Science and Engineering

Tokyo, Japan

$M$ Dojama, CAMSE ' 90 Nikan

Kogyo Shimbun Ltd., Business

Bureau, 8-10 Kudan Kita, 1-

chome, Chiyoda-ku, Tokyo 102 ,

Japan; (03) 222-7162; fax (03)

221-7137

\section{SEPTEMBER 1990}

37

6th Europhysics Top Conference

Lattice Defects in lonic Materials

Groningen, The Netherlands

H.W. den Hartog, Solid Physics

Lab, Melkweg 1, NL-9718 EP

Groningen

5-7

Analysis by a Combination of lon

Beam (Accelerator-Based) and

Surface Specific Techniques

Namur, Belgium

G. Demortier, Département de

Physique, Facultes Universitaires

N-D de la Paix, nue de Brocelles.

61. B-5000 Narmur Belgium; tax 32-81-2303 91

M[RS Ion Beam Modification of

Materials-IBMA '90

Knoxville, TN

P.H. Green, IBMM '90, Oak Ridge

National Lab., Solid State Div.

P.O. Box 2008, M.S.6033, Oak

Ridge, TN 37831-6033;

(615) $576-1864$; tax (615)

$574-4143$

(See related articye in

Vb. XVNo. 12.)

10-13

2nd World Congress on Super-

conductivity

Houston, TX

World Congress on Super-

conductivity, P.O. Box 27805

Houston, TX 77227-7805; (713)

$895-2500$; $\operatorname{tax}(713) 623-3560$

10-14

2nd Intemational Conterence on

Plasma Surface Engineering

Garmisch-Partenkirchen, W.

Germany Deutsche Gesellschaft

ur Gavano-u Oberfïchentech

rik e. V. Horionplatz 6, D-4000

Dusseldort 1, W. Gemany; 0211/

132381

$10-20$

NAIT Advanced Study Institute 
24-27

European Gallium Arsenide

Conference

St. Helier, Jersey, Channel islands

Meetings Officer, Institute of

Ptysics, 47 Belgrade Square.

London SW1X 80X, England

24-28

1990 Applied Superconductivity

Conference

Aspen, $\mathrm{CO}$

ASC190, Centennial Conterences,

5353 Manhattan Circle, Suite 103

Boulder, 0080303 ; (303)

4992299

\section{OCTOBER 1990}

36

American Society of Composites: Fourth Technical Conference on Composite Materials

Blacksburg, VA

M.W. Hyer, RSM Dept., Virginia

Tech., Blacksburg, VA 24061;

(703) 231-5372

$7-11$

TMS Fall Meeting: Physical

Metallurgy and Materials

Detroit, MI

TMS, 420 Commonwealth Dr.

Warrendale, PA 15086; (412) 776 -

9050; $\operatorname{tax}(412) 7763770$

8-12

37th National Vacuum

Symposium

Toronto, Ontario, Canada

M. Churchill, American Vacuum

Society, 335E. 45th St. New

York, NY 10017; (212) 661-9404

1419

178th Meeting of The Electro-

chemical Society

Seattle. WA

The Electrochemical Society, 10

S. Main St., Pennington, NJ

085342896

15-17

ISHM '90: Intemational Sympo-

sium on Microelectronics

Chicago, IL

N. Binkley, ISHM, P.O. Box 2698,

Reston, VA 22090-2698; (703)

471-0066; $\operatorname{tax}(703)$ 471-1937

21-24

11 th Intemational Workshop on

Rare-Earth Magnets and Their

Applications

Pitsburgh, PA

S.G. Sankar, Carnegie Mellon

University, Mellon Institute, $\mathbf{4 4 0 0}$

Fifth Ave., Pittsburgh, PA 15213

(412) 268-5649; $\operatorname{tax}(412) 268$

3101
25

6th Intemational Symposium on Magnetic Anisotropy and

Coercivity in Rare-Earth-Transition Metal Alloys

Pittsburgh, PA

S.G. Sankar Camegie Melton

University, Melbn Institute, $\mathbf{4 4 0 0}$

Fifth Ave., Pittsburgh, PA 15213;

(412) 268-5649; tax (412) 268-

3101

$27-2$

Intemational Conference on

Physical Concepts of Materials for Novel Optoelectronic Device

Appications

Aachen, W. Germany

SPIE, P.O. BOx 10, Bellingham,

WA 98227-0010; (206) 676-3290;

$\operatorname{tax}(206) 647-1445$

28-31

TMS Fall Meeting: Extractive \&

Process Metallurgy

Williamsburg. VA

TMS, 420 Commonwealth Dr.

Warrendale, PA 15086; (412) 776 9050; tax (4†2) 776-3770

\section{NOVEMBER 1990}

49

OPTCON ' 90

Boston MA

SPIE, P.O. Box 10, Bellingham,

WA 98227-0010; (206) 676-3290;

$\operatorname{tax}(206)$ 647-1445

4-9

Lasers and Electro-Optics Society

Annual Meeting (in conil

with OPTCON' 90 )

Boston, MA

作 445 Hoes Lane. P.O. Box 1331.

Piscataway, NJ 08855-1331; (201) 562-3895; $\operatorname{tax}$ (201) 981-0027

58

11th Intemational Conference on the Application of Accelerators in

Research and Industry

Denton, TX

J.L. Duggan, University of North

Texas, Dept. of Physics, P.O. Box

5368, Denton, TX 76203; (817) 565-3252

6-9

4th Intemational Surface

Modification Conference

Paris, France

Societé Francais de Métallurgie. Immeuble Elysíes La Détense, 19. Le ParvisCedex 35,92072,

Paris La Défense, France; (1) 47

678871 ; fax (1) 47678577 ;

TMS, 420 Commonwealth Dr.

Warmendale, PA 15086; (412) 776

9050; $\operatorname{tax}(412) 776-3770$
Nuctéaires, Laboratoire PHASE

$13-15$

2nd Intemational Ceramic Science and Tectinology Congress

Orlando F

American Ceramic Society, 757

Brooksedge Plaza Drive, Wester-

(614) 890-4700;

$\operatorname{tax}(614) 8996109$

13-15

ACerS Electronics Division

Meeting

Orlando, FL

American Ceramic Society, 757

Brooksedge Plaza Drive, Wester-

ville, OH 43081; (614) 890-4700;

$\operatorname{tax}(614) 8996109$

$26-1$

MIRIS Matertak Research

Sociely Fall Merting

Boston, MA

M. Geil, Materials Research

Society, 9800 McKnight Rd.

Pittsburgh, PA 15237; (412) 367 -

3003; $\operatorname{tax}(412) 367-4373$

26-7

Experimental Workshop on HighTemperature Superconductors and Related Materials (Advanced

Activities)

Trieste, ltaly

Intemational Center for Theoretical Ptysics, P.0. Box 586,

34100, Trieste, Italy; 22401; fax 224163

$27-30$

\section{EATRS European Matertats}

Strashourn France

P. Siffert, Centre de Recherches

France; (88) 28 65 43; $\operatorname{tax}(88) 28$ 0990

\section{FEBRUARY 1991}

10-15

POLYMER 91-Polymer Materi-

as: Preparation, Characterization

and Properties

Melboume, Australia

G.B. Guises, RAC Polymer Div

P.O. Box 224, Belmont, Victoria

3216 Australia; 05247 2625; tax

05247265

$16-22$

OFC-Optical Fibers Communica

tions Conference

San Diego, CA

Lasers and Electro-Optics Society.

445 Hoes Lane, P.O. Box 1331.

Piscataway, NN 08855-1331; (201)

562-3895; fax (201) 981-0027
$17-21$

TMS Annual Meeting

New Ordeans, LA

TMS, 420 Commonwealth Dr.

Warrendale, PA 15086; (412) 776

9050; $\operatorname{tax}(412) 776-3770$

\section{MARCH 1991}

18-22

American Physical Society

Meeting

Cincinnati, $\mathrm{OH}$

APS, 335 E. 45th St., New York

NY 10017; (212) 682-7341

\section{APAIL 1991}

7-11

Intemational Conference on

Wear of Materiats-91

Orlando, $\mathrm{FL}$

L. Friedman, Meetings Dept.,

ASME, 345 E. 47th St., New

York. NY 10017

(See related articte in

Vo. XVNo. 12.)

15-17

Frontiers of Tribology

Strattord Upon Avon, United

Kingdom

Meetings Office, Institute of

Physios, 47 Belgrave Square.

Landon SW1X 80X-01-235-6111

16-18

Magneto-Optical Recording

Intemational Symposium '91

Tokyo, Japan

T. Surauk, IBM Almaden Research

Center, 650 Harry Rd., San Jose,

CA 95120-6099; (408) 927-2099;

tax (408) $927-2100$

MAY 1991

5-11

3rd European Conference on

Crystal Growth

Budapest, Hungary

E. Lentvay Research Inst. for

Techrical Physics, Hungarian

Acad. of Science, Ujpest 1, PF.

76. Budapest, Hungary 1325:

(36) (1) $698-037$

13-17

CLEO '91: Conference on Lasers and Electuo-Optics

Baltimore, MD

Lasers and Electro-Optics Society.

445 Hoes Lane PO Box 1331

Piscataway, NJ 08855-1331; (201)

562-3896; tax (201) 981-0027

JUNE 1991

$17-20$

6th Japan Institute of Metals

Symposium on Intermetallic

Compounds

Sendai, Japan

0. Lami, Institute for Materials

Research, Tohoku Univ., Katahira,

Sendai 980 Japan; (022) 227

6200; tax (022) 264-7984

$18-2$

Electronic Materials Conference

Boulder, CO

TMS, 420 Commomwealth Dr.

Warrendale, PA 15086; (412) 776

9050; $\operatorname{tax}(412) 776-3770$

JULY 1991

21-26

American Cystallographic

Association Annual Meeting

Toledo, $\mathrm{OH}$

M.C. Etter Dept. of Chemistry

Univ. of Minnesota, 78 Kolthoff

Hall, Minneapolis, MN 55455;

(612) 6245217

SEPTEMBER 1991

17-19

2nd Intemational Conterence on

Interfacial Phenomena in

Composite Materiaks ' 91

Lerven, Belgium

1 Miles, Butterworth Scientific

Ltd., P.O. Box 63, Westbury

House, Bury St., Guildford.

Surrey GU2 58H United Kingdom;

0483 300066; t tax 0483301563

$30-2$

Specialty Polymers '91: Supramolecular Aspects of Polymer

Synthesis and Polymer Structure

Mainz, W. Germany 